\title{
The strange degrees of freedom in QCD at high temperature
}

\author{
Christian Schmidt* (for RBC-Bielefeld Collaboration) \\ Universität Bielefeld, Fakultät für Physik, Postfach 100131, D-33501 Bielefeld, Germany \\ E-mail: schmidtephysik. uni-bielefeld.de
}

\begin{abstract}
We discuss recent results on fluctuations of conserved charges, and their approach to the hadron resonance gas at temperatures below the chiral crossover $\left(T_{c}\right)$ as well as to a gas of free quarks at very high temperatures. We will focus on the strange degrees of freedom and verify that they are consitent with those of an uncorrelated gas of hadrons for temperatures of $T \lesssim 160 \mathrm{MeV}$ and that they can be discribed by a quasi particle model at best for $T \gtrsim 2 T_{c}$. To extract this information we use all cumulants of net baryon number and net strangeness fluctuations and their correlations up to the fourth order. In particular we propose observables that serve as indicator for the validity of the hadronic degrees of freedom and show that the partial pressures from different strangeness sectors agree separately with HRG model predicions.
\end{abstract}

31st International Symposium on Lattice Field Theory - LATTICE 2013

July 29 - August 3, 2013

Mainz, Germany

\footnotetext{
* Speaker.

${ }^{\dagger} \mathrm{CS}$ acknowledges support by the BMBF under grant 05P12PBCTA, the numerical calculations have been performed using the USQCD GPU-clusters at JLab, the Bielefeld GPU cluster and the NYBlue at the NYCCS
} 


\section{Introduction}

The fluctuations of conserved charges in hot and dense QCD carry a variety of information that can be used to probe thermodynamic properties of QCD. These quantities can be obtained as derivatives of the QCD partition function with respect to appropriate chemical potentials. The original motivation to study these quantities stems from the idea of calculating successive orders in a Taylor expansion of the pressure with respect to a baryon chemical potential [1]. As direct lattice QCD calculations are harmed by the notorious sign problem, a Taylor expansion provides one possibility to obtain corrections to bulk thermodynamic quantities that are due to the presence of a dense medium, in a rigorous and controled manner. Moreover, the convergence radius of such expansions can provide valuable information on the existence or non-existence of a critical point in the QCD phase diagram [2,3]. Secondly, cumulants of fluctuations of conserved charges can also be measured experimentally in ultra relativistic heavy ion collisions (HICs). They are excellent indicators for QCD critical behavior [4] and thus important observables in the context of a critical point search. In fact, recently large experimental effort is made, e.g. as part of the RHIC beam energy scan (BES) [5, 6], to determine higher order cumulants of multiplicity fluctuations. During the evolution of a HIC, a point is reached where particle multiplicities and their fluctuations freezeout, once the fireball that is created by the colliding nuclei, has sufficiently cooled and expanded. Matching (lattice) QCD calculations and experimental measurements on fluctuations, one is able to estimate external thermodynamic parameters, such as temperature and chemical potential, that are associated with the freeze-out point. [7].

Finally — and this is the motivation that builds the basis of this study — cumulants of conserved charge fluctuations reveal information of the elementary units of charges that are carried by the effective degrees of freedom in the system [8]. With other words, the assumption that the quantum numbers of the effective degrees of freedom in the system are those of hadrons will lead to very different predictions for the fluctuations than those one would get from a gas of free quarks. For our lattice calculations we use $2+1$ flavor of highly improved staggered quarks (HISQ) [9] with almost physical masses, i.e. the light quark mass $\left(m_{l}\right)$ has been chosen as $m_{l}=m_{s} / 20$, while we tuned the strange quark mass $\left(m_{s}\right)$ to its physical value. This corresponds to a Goldstone Pion mass of $m_{\pi} \approx 160 \mathrm{MeV}$. We will compare our lattice QCD results on fluctuations to the hadron resonance gas (HRG) model at low temperatures and to quasi free quarks at high temperatures. Here we focus on net strangeness fluctuations and their correlations with net baryon number. As its mass is of the order of the temperatures of interest, the strange quark always played a special role in the analysis of the quark gluon plasma, in both experimental and theoretical studies [10]. Furthermore, the possibility of a flavor specific hierarchy of deconfinement has been discussed recently [11], triggered by rather large values of the transition temperature, determined from Polyakov Loop and net strangeness fluctuations [12]. We do not find a clear signal for such hierarchy in this study [13].

\section{Definition and results of cumulants}

In thermodynamics, the study of susceptibilities, i.e. derivatives of the logarithm of the partition function with respect to external parameter, is a widely used concept, as the calculation of the partition function itself is in many cases rather cumbersome. In the framework of lattice regular- 

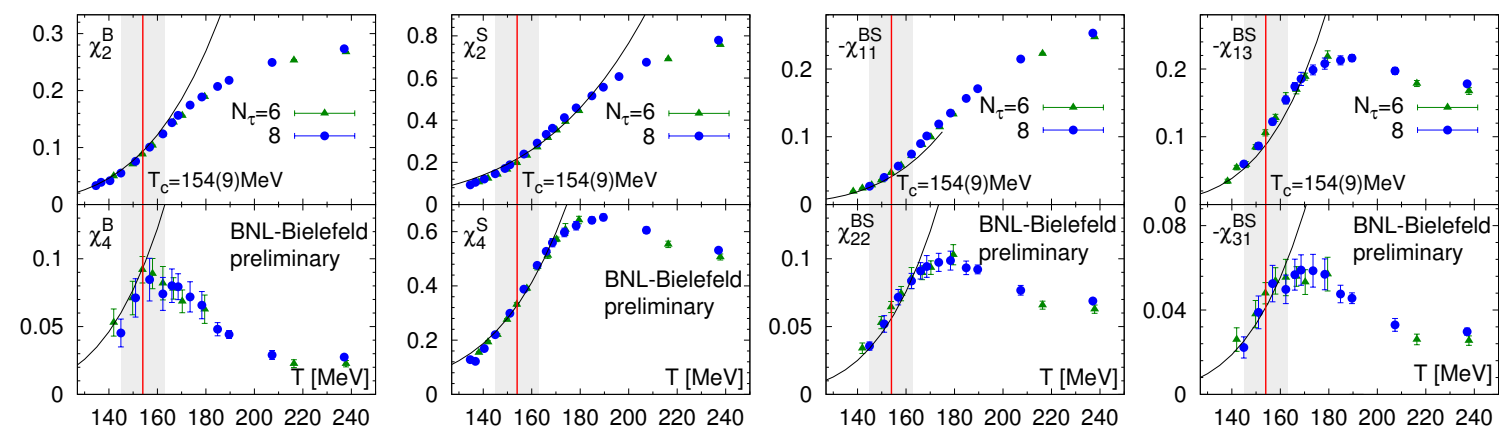

Figure 1: Diagonal cumulants of net baryon number and net strangeness fluctuations and their off-diagonal correlations up to 4th order as a function of temperature. Vertical bands indicate the chiral crossover temperature [14]. The corresponding results from the HRG model are shown as solid black lines.

ized QCD, cumulants of the fluctuations of conserved charges, such as net baryon number (B), net strangeness (S) or net electric charge $(\mathrm{Q})$, can be readily obtained as such (generalized) susceptibilities as they are defined as derivatives with respect to the chemical potentials of the conserved charges $\mu_{X}$ with $X=B, Q, S$. The diagonal cumulants and their off-diagonal correlations can be defined as

$$
\chi_{m n l}^{B Q S}=\left.\frac{\partial^{(m+n+l)}\left[P\left(T, \hat{\mu}_{B}, \hat{\mu}_{Q}, \hat{\mu}_{S}\right) / T^{4}\right]}{\partial \hat{\mu}_{B}^{m} \partial \hat{\mu}_{Q}^{n} \partial \hat{\mu}_{S}^{l}}\right|_{\vec{\mu}=0},
$$

where $P$ denotes the pressure ${ }^{1}$ and $\hat{\mu}_{X}=\mu_{X} / T$. In the following we will drop pairs of upper and lower indices in case the lower index vanish identical, i.e. we follow the convention $\chi_{101}^{B Q S} \equiv \chi_{11}^{B S}$ and so forth. Experimentally, the cumulants are determined from corresponding net charge fluctuations around their central values. In Fig. 1 we show our lattice results for all net baryon number and net strangeness cumulants and their correlations up to fourth order, obtained from $\mathscr{O}\left(10^{4}-10^{5}\right)$ lattice configurations per temperature. A more detailed description of the necessary operators that have to be measures can be found, e.g., in [15]. Also shown are the corresponding results from the HRG model, that will be discussed in more detail in Sec. 3. In general we find good agreement with the HRG model for temperatures $T \lesssim 160 \mathrm{MeV}$.

\section{The hadronic phase}

A widely used model for the hadronic phase of QCD is the HRG model. Here we use the most simplistic version where the total pressure is given as sum over all partial pressures that are obtained by the free quantum gases of all particles and resonances from the particle data booklet, up to a mass cut-off of $2.5 \mathrm{GeV}$. I.e., no interactions (for instance hard core repulsion) are taken into account. In fact, the interactions between hadrons are already included through the appearance of resonances [16]. Such type of models are used very successfully for the description of experimentally measured particle multiplicities and their fluctuations [17] and also yield important guiding for bulk thermodynamic observables from the lattice [18, 19]. From Fig. 1 it is already clear that the HRG model leads to an excellent description also for the observables presented here.

\footnotetext{
${ }^{1}$ In the grand canonical ensemble we have $P=T \ln Z / V$
} 

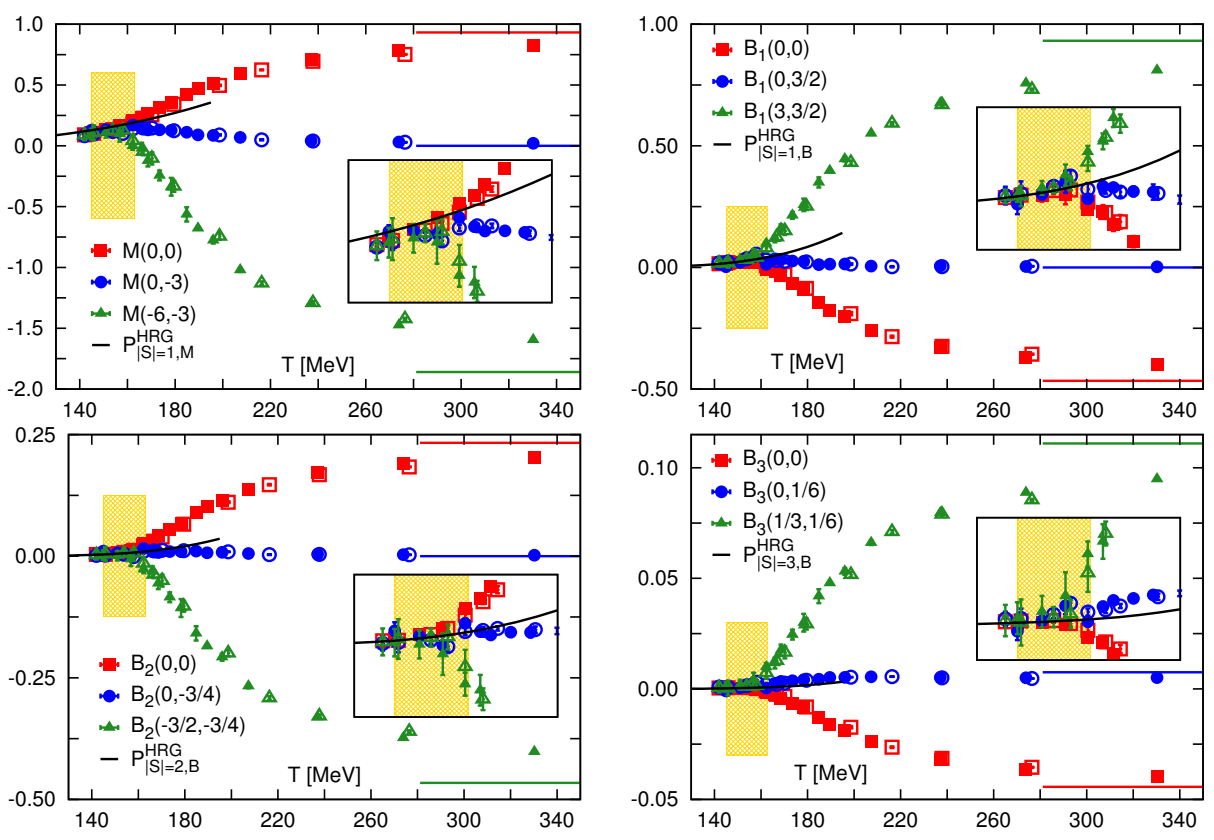

Figure 2: Operators that project for low temperatures onto the partial pressure of strange mesons (top-left), baryons with strangeness $|S|=1$ (top-right), $|S|=2$ (bottom-left) and $|S|=3$ (bottom-right). Results from $N_{\tau}=6$ and 8 lattices are shown by open and full symbols, respectively. Also shown are results from the HRG model (black solid lines). Vertical bands indicate the chiral crossover temperature [14].

This statement can, however, be further refined. Firstly, we can check whether the agreement with the HRG is valid within separate strangeness sectors. Secondly, we can construct observables that vanish independent of temperature (up to corrections to Boltzmann statistics) as soon as the HRG yields a valid description of the system. Those quantities provide an optimal measure for the validity of the HRG model. Within the HRG model the pressure that stem from strange particles receives contributions from 4 different sectors,

$$
\begin{aligned}
P_{S}^{\mathrm{HRG}}\left(T, \hat{\mu}_{B}, \hat{\mu}_{S}\right) & =P_{|S|=1, M}^{\mathrm{HRG}}(T) \cosh \left(\hat{\mu}_{S}\right)+P_{|S|=1, B}^{H R G}(T) \cosh \left(\hat{\mu}_{B}-\hat{\mu}_{S}\right) \\
& +P_{|S|=2, B}^{H R G}(T) \cosh \left(\hat{\mu}_{B}-2 \hat{\mu}_{S}\right)+P_{|S|=3, B}^{H R G}(T) \cosh \left(\hat{\mu}_{B}-3 \hat{\mu}_{S}\right),
\end{aligned}
$$

where $P_{|S|=i, M / B}^{H R G}$ denotes the partial pressure from mesons $(M)$ or baryons $(B)$ with strangeness $|S|=i$. We also assume Boltzmann statistics here, which leads to the factorization of the $\mu$ dependence ${ }^{2}$. Eq. (3.1) immediately leads to linear relations between the quantities defined through Eq. (3.1) (shown in Fig. 1) and the partial pressures from different strangeness sectors. Inverting these relations enables us to project to the partial pressures. we denote the projectors as $M_{1}, B_{1}, B_{2}$ and $B_{3}$, which we plot in Fig. 2 Note that the projectors are not defined uniquely, as each of it depends on two parameter $c_{1}, c_{2}$. However, as long as the HRG is valid, the dependence on $c_{1}$ and $c_{2}$ should vanish. This is seen in Fig. 2 where we plot each of the projectors for 3 choices of the parameters. We find indeed, that for $T \lesssim 160 \mathrm{MeV}$ the dependence on $c_{1}$ and $c_{2}$ vanish and the

\footnotetext{
${ }^{2}$ The validity of Boltzmann approximation is ensured here, since for the lightest strange hadron (Kaon) we have $m / T \gtrsim 3$ for the temperature values considered here.
} 


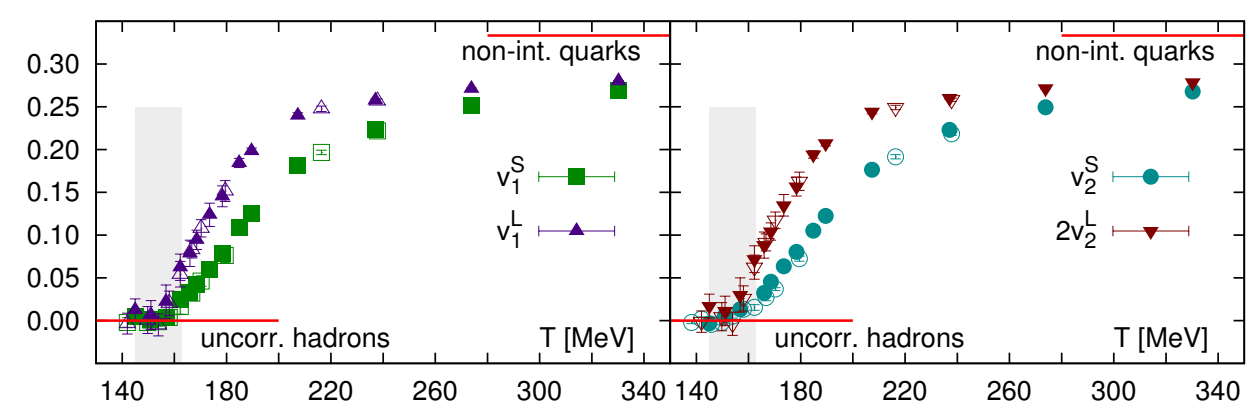

Figure 3: Quantities that vanish within and uncorrelated gas of hadrons (Boltzmann approximation).

results agree with each other and also with the partial pressure of the specific strange sector that is computed from the HRG model. The 2-dim. freedom in the definition of the projectors originates from the 2-dim. null space of the linear mapping between our 6 basis observables shown in Fig. 1 and the partial pressures from the 4 strangeness sectors. As basis vectors of this null space we can choose the linear combinations $v_{1}^{S}=\chi_{31}^{B S}-\chi_{11}^{B S}$ and $v_{2}^{S}=\frac{1}{3}\left(\chi_{2}^{S}-\chi_{4}^{S}\right)-2 \chi_{13}^{B S}-4 \chi_{22}^{B S}-2 \chi_{31}^{B S}$ [13]. Again, these combinations of cumulants vanish in the HRG model (up to corrections to the Boltzmann statistics) and provide a measure for the quality of the HRG model. As $v_{1}^{S}$ and $v_{2}^{S}$ receive contributions from strange hadrons only, they also indicate the temperature from where on strange quarks start to be liberated into the system. The whole analysis can be repeated for correlations of the net baryon number with other flavors as proposed in [11]. In Fig. 3 we plot the susceptibilities $v_{1}^{S}, v_{2}^{S}$ together with $v_{1}^{L}, v_{2}^{L}$, where $L$ indicates the net lightness number. In analogy to Eq. (2.1) we define $\chi_{n}^{L}$ by $\chi_{n}^{L}=\partial^{n}\left(p / T^{4}\right) / \partial \hat{\mu}_{L}^{n}$ and $\partial \hat{\mu}_{L}=\left(\partial \hat{\mu}_{u}+\partial \hat{\mu}_{d}\right) / 2$ where $\mu_{u}$ and $\mu_{d}$ are up and down quark chemical potentials, respectively. In contrast to $v_{1}^{S}$ and $v_{2}^{S}, v_{1}^{L}$ and $v_{2}^{L}$ receive also contributions from protons and neutrons and thus can be understood as a more general deconfinement observable. As can be seen from Fig. 3 their behavior is very similar. Especially the temperature at which the observables deviate from zero is similar. We thus conclude that within our current accuracy strange hadrons do not dissolve at larger temperatures than light hadrons.

\section{The plasma phase}

At very high temperatures we expect to find quantum numbers of the effective degrees of freedom that resemble those of quarks. If the strange degrees of freedom can be described by a weakly interacting gas of quasi-quarks, then strangeness is associated with a fractional baryon number of $B=1 / 3$ and a electric charge of $Q=-1 / 3$. For correlations of net strangeness with net baryon number and net electric charge on thus obtains

$$
\frac{\chi_{m n}^{B S}}{\chi_{m+n}^{S}}=\frac{(-1)^{n}}{3^{m}} \quad \frac{\chi_{m n}^{Q S}}{\chi_{m+n}^{S}}=\frac{(-1)^{m+n}}{3^{m}},
$$

where $n, m>0$ and $m+n=2,4$. In Fig. 4 we show our results for these ratios, scaled by the propper powers of fractional baryonic and electric charges. The shaded regions at high temperatures indicate the range of values for these ratios as predicted for weakly interacting quasi-quarks from 


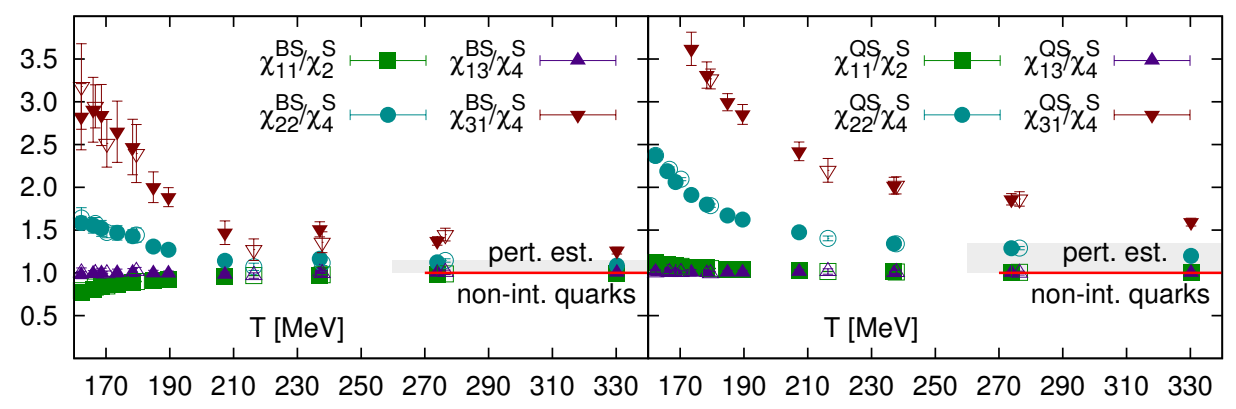

Figure 4: Baryon number strangeness (left) and electric charge strangeness correlations (right), properly scaled by the diagonal strangeness cumulants and powers of the fractional baryonic and electric charges [see Eq. (4.1)]. In the non-interacting massless quark gas all these observables are unity (shown by the lines at high temperatures). The shaded regions indicate the range of perturbative estimates (see text) for all these observables obtained using one-loop re-summed HTL calculations [20]. Results from $N_{\tau}=6$ and 8 lattices are shown by open and filled symbols, respectively.

the re-summed Hard Thermal Loop (HTL) perturbation theory at the one-loop order [20], using one-loop running coupling obtained at the scale between $\pi T$ and $4 \pi T$. We find that second order ratios are much closer to those expected for weakly interacting quasi-quarks, differing at a few percent level for $T \sim 1.25 T_{c}$. Previous lattice QCD calculations showed similar results [19, 21, 22], suggesting that the strange degrees of freedom in the QGP can be described by weakly interacting quasi-quarks even down to temperatures very close to $T_{c}$. However, our results involving correlations of strangeness with higher powers of baryon number and electric charge clearly indicate that a description in terms of weakly interacting quasi-quarks can only be valid for temperatures $T \gtrsim 2 T_{c}$. Note, that the HTL perturbative expansion for ratios involving one derivative w.r.t the baryonic/electric chemical potential $\left(\chi_{11}^{X S} / \chi_{2}^{S}\right.$ and $\left.\chi_{13}^{X S} / \chi_{4}^{S}, X=B, Q\right)$ starts differing from the noninteracting quark gas limit at $\mathscr{O}\left(\alpha_{s}^{3} \ln \alpha_{s}\right)$ [23]. For ratios involving higher derivatives w.r.t the baryonic/electric chemical potentials $\left(\chi_{22}^{X S} / \chi_{4}^{S}\right.$ and $\left.\chi_{31}^{X S} / \chi_{4}^{S}, X=B, Q\right)$ the difference starts at $\mathscr{O}\left(\alpha_{s}^{3 / 2}\right)$ [20]. Here $\alpha_{s}$ denotes the strong coupling constant. The enhancement of the higher order baryon number strangeness and electric charge strangeness correlations is thus likely to be expected within the regime of the validity of the weak coupling expansion.

\section{References}

[1] C. R. Allton, S. Ejiri, S. J. Hands, O. Kaczmarek, F. Karsch, E. Laermann, C. Schmidt and L. Scorzato, Phys. Rev. D 66 (2002) 074507 [hep-lat/0204010].

[2] C. Schmidt, Prog. Theor. Phys. Suppl. 186 (2010) 563 [arXiv:1007.5164 [hep-lat]].

[3] R. V. Gavai and S. Gupta, Phys. Rev. D 71 (2005) 114014 [hep-lat/0412035].

[4] M. A. Stephanov, Phys. Rev. Lett. 102 (2009) 032301 [arXiv:0809.3450 [hep-ph]].

[5] L. Adamczyk et al. [STAR Collaboration], arXiv:1309.5681 [nucl-ex].

[6] P. Garg [for PHENIX Collaboration], PoS CPOD 2013 (2013) 050 [arXiv:1305.7327 [nucl-ex]]. arXiv:1309.5681 [nucl-ex]. 
[7] A. Bazavov, H. T. Ding, P. Hegde, O. Kaczmarek, F. Karsch, E. Laermann, S. Mukherjee and P. Petreczky et al., Phys. Rev. Lett. 109 (2012) 192302 [arXiv:1208.1220 [hep-lat]].

[8] S. Ejiri, F. Karsch and K. Redlich, Phys. Lett. B 633 (2006) 275 [hep-ph/0509051].

[9] E. Follana et al. [HPQCD and UKQCD Collaborations], Phys. Rev. D 75 (2007) 054502 [hep-lat/0610092].

[10] For recent reviews see: B. V. Jacak and B. Muller, Science 337, 310 (2012); B. Jacak and P. Steinberg, Phys. Today 63N5, 39 (2010).

[11] C. Ratti, R. Bellwied, M. Cristoforetti and M. Barbaro, Phys. Rev. D 85, 014004 (2012); R. Bellwied, S. Borsanyi, Z. Fodor, S. DKatz and C. Ratti, arXiv:1305.6297 [hep-lat].

[12] Y. Aoki, Z. Fodor, S. D. Katz and K. K. Szabo, Phys. Lett. B 643, 46 (2006); Y. Aoki et al., JHEP 0906, 088 (2009); S. Borsanyi et al. [Wuppertal-Budapest Collaboration], JHEP 1009, 073 (2010);

[13] A. Bazavov, H. -T. Ding, P. Hegde, O. Kaczmarek, F. Karsch, E. Laermann, Y. Maezawa and S. Mukherjee et al., Phys. Rev. Lett. 111, 082301 (2013) [arXiv:1304.7220 [hep-lat]].

[14] A. Bazavov, T. Bhattacharya, M. Cheng, C. DeTar, H. T. Ding, S. Gottlieb, R. Gupta and P. Hegde et al., Phys. Rev. D 85 (2012) 054503 [arXiv:1111.1710 [hep-lat]].

[15] C. R. Allton, M. Doring, S. Ejiri, S. J. Hands, O. Kaczmarek, F. Karsch, E. Laermann and K. Redlich, Phys. Rev. D 71 (2005) 054508 [hep-lat/0501030].

[16] R. Dashen, S. -K. Ma and H. J. Bernstein, Phys. Rev. 187 (1969) 345.

[17] P. Braun-Munzinger, K. Redlich and J. Stachel, In *Hwa, R.C. (ed.) et al.: Quark gluon plasma* 491-599 [nucl-th/0304013].

[18] M. Cheng, S. Ejiri, P. Hegde, F. Karsch, O. Kaczmarek, E. Laermann, R. D. Mawhinney and C. Miao et al., Phys. Rev. D 81 (2010) 054504 [arXiv:0911.2215 [hep-lat]].

[19] A. Bazavov et al. [HotQCD Collaboration], Phys. Rev. D 86 (2012) 034509 [arXiv:1203.0784 [hep-lat]].

[20] J. O. Andersen, S. Mogliacci, N. Su and A. Vuorinen, Phys. Rev. D 87 (2013) 074003 [arXiv:1210.0912 [hep-ph]].

[21] S. Borsanyi, Z. Fodor, S. D. Katz, S. Krieg, C. Ratti and K. Szabo, JHEP 1201 (2012) 138 [arXiv:1112.4416 [hep-lat]].

[22] M. Cheng, P. Hendge, C. Jung, F. Karsch, O. Kaczmarek, E. Laermann, R. D. Mawhinney and C. Miao et al., Phys. Rev. D 79 (2009) 074505 [arXiv:0811.1006 [hep-lat]].

[23] J.-P. Blaizot, E. Iancu and A. Rebhan, Phys. Lett. B 523 (2001) 143. 